\title{
Localized phase structures growing out of quantum fluctuations in a quench of tunnel-coupled atomic condensates
}

\author{
Clemens Neuenhahn, ${ }^{1, \text { * }}$ Anatoli Polkovnikov, ${ }^{2}$ and Florian Marquardt ${ }^{1}$ \\ ${ }^{1}$ Friedrich-Alexander-Universität Erlangen-Nürnberg, \\ Institute for Theoretical Physics II, Staudtstr. 7, 91058 Erlangen, Germany \\ ${ }^{2}$ Department of Physics, Boston University, 590 Commonwealth Avenue, Boston, Massachusetts 02215, USA
}

\begin{abstract}
We investigate the relative phase between two weakly interacting 1D condensates of bosonic atoms after suddenly switching on the tunnel-coupling. The following phase dynamics is governed by the quantum sine-Gordon equation. In the semiclassical limit of weak interactions, we observe the parametric amplification of quantum fluctuations leading to the formation of breathers with a finite lifetime. The typical lifetime and density of the these 'quasibreathers' are derived employing exact solutions of the classical sine-Gordon equation. Both depend on the initial relative phase between the condensates, which is considered as a tunable parameter.
\end{abstract}

Dynamical instabilities can amplify spatial field fluctuations drastically. If the instability provides sufficient energy, even quantum zero-point fluctuations can trigger the formation of macroscopic field patterns. For instance, in some cosmological scenarios of the inflationary stage of the universe, following a slow-roll, a scalar inflaton field performs oscillations around the minimum of the corresponding inflaton potential. Thereby, resonant spatial fluctuations are amplified parametrically. For a rather generic class of potentials these can end in longlived, local concentrations of energy, so-called 'oscillons' (e.g., [1-4]).

In this work, we argue that analogous non-equilibrium phenomena should be observable in experiments with a pair of weakly interacting quasi-1D clouds of cold, bosonic atoms [5, 6]. After suddenly turning on the tunnel coupling between the condensates, the dynamics of the relative phase field $\hat{\phi}$ is governed by the integrable quantum sine-Gordon model [7]

$$
\frac{d^{2} \hat{\phi}}{d t^{2}}-\frac{d^{2} \hat{\phi}}{d x^{2}}+\frac{m^{2}}{\beta} \sin \beta \hat{\phi}=0,
$$

where the 'mass' $m$ depends on the tunnel amplitude, such that $m(t)=m \Theta(t)$ for this quench. The phase has been rescaled, and for weak interactions $\beta \ll 1$.

The sine-Gordon model (SGM) is one of the most prominent prototypical models of low-dimensional condensed-matter systems. Currently, quenches in the SGM are under intense investigation (e.g., 8111). For instance, only recently the amplification of density inhomogeneities after a quench to the special Luther-Emmery point $\left(\beta^{2}=4 \pi\right)$, was demonstrated [12].

Here, we propose that the spatially averaged value $\Phi$ of the relative phase field is tuned to some value $\Phi_{0}$ right before the quench (e.g., this might be achieved by slightly tilting the transversal double-well potential confining the BECs, cf. Fig.17). The subsequent phase dynamics can be directly observed in matter wave interference experiments [5, 6].

At short times, $\Phi$ will perform Josephson oscillations (Fig.1b) according to $d^{2} \Phi / d t^{2}=-\beta^{-1} m^{2} \sin \beta \Phi$. How-

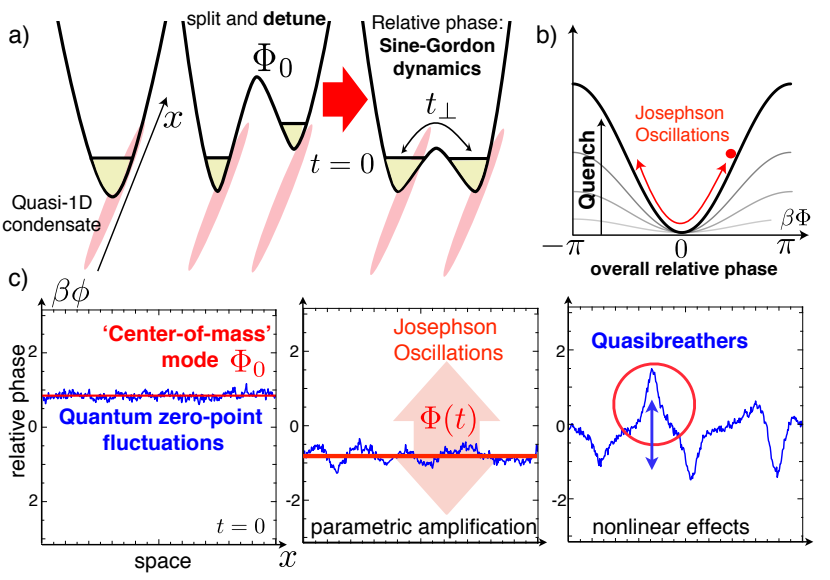

Figure 1. a) Proposed experimental protocol. A single quasi$1 \mathrm{D}$ condensate is split and the global phase is tuned to $\Phi_{0}$. Switching on the tunnel-coupling, the dynamics of the relative phase obeys the quantum sine-Gordon equation. b) At short times, the global phase performs Josephson oscillations c) Single run of TWA (see main text) with $\beta \Phi_{0}=0.25 \pi$. Spatial quantum fluctuations are amplified parametrically. Eventually, the non-linearity of the sine-Gordon equation kicks in and 'quasibreathers' (breathers with a finite lifetime) form.

ever, these are linearly unstable [13, 14] in the presence of inhomogeneous quantum fluctuations which are parametrically amplified at certain wave lengths (Fig.1p). As we will show in this paper, due to this modulation instability at some later point the dynamics becomes fully nonlinear and one observes the formation of sharply localized and oscillating patterns in the phase-field (Fig.1). It will be shown below that they can be related to particular exact solutions of the classical SGM obtained from the Bäcklund transformation (following [15]). These 'quasibreathers' (QBs) have a finite lifetime (in contrast to the well-known breather solutions), and we find good evidence that a quasi-equilibrium steady-state with a finite density of such excitations develops at long times. 
For the considered quench, due to the intrinsic instability, a simple approximation of the SGM in terms of non-interacting, massive phonons is invalid, even though $\beta \ll 1$. Instead, we employ the truncated Wignerapproximation (e.g., [16]) (TWA). The basic idea behind TWA is to simulate classical field equations, but with quantum-mechanical fluctuations as stochastic initial conditions. In the limit of $\beta \rightarrow 0$, TWA is expected to become reliable as it can be shown that $\beta$ plays the role of an effective Planck's constant [16]. It describes correctly the linear dynamics during the parametric amplification. Once QBs form, occupation numbers are already large such that a semiclassical description (provided by TWA) should continue to remain valid. The great advantage of TWA is that it serves snapshots (Fig.1 1 $)$ of the phase field $\phi(x, t)$ run-by-run, which can be compared directly to the experimental observations.

In the following, we introduce the model and demonstrate that the proposed setup should be well within the reach of present experiments. After a discussion of the numerical findings obtained from TWA, we will introduce the analytical 'quasibreather' solutions of the classical SGM. We demonstrate that these solutions are well suited to explain the main physical features as predicted by TWA. Eventually, we argue that a statistical analysis of $\phi(x, t)$, experimentally obtained at a single time $t$ per run, could reveal the distinctive signature of QBs.

Model. - It was shown [7 that on scales larger than the condensate healing length $\xi_{h}$, the dynamics of the relative phase between condensates $\hat{\phi} \equiv\left(\hat{\phi}_{1}-\hat{\phi}_{2}\right) / \sqrt{2}$ (and of the density field $\hat{\Pi}$, fulfilling $\left.\left[\hat{\Pi}(x), \hat{\phi}\left(x^{\prime}\right)\right]=i \hbar \delta\left(x-x^{\prime}\right)\right)$ is governed by the quantum SGM. In the following, we use the notation in [7]. After rescaling the fields $(\hat{\phi} \mapsto$ $\sqrt{\pi / K} \hat{\phi}$ and $\hat{\Pi} \mapsto \sqrt{K / \pi} \hat{\Pi})$, the Hamiltonian reads

$$
\hat{H}=\frac{\hbar v_{s}}{2} \int d x\left[\hat{\Pi}^{2}+\left(\partial_{x} \hat{\phi}\right)^{2}\right]-\frac{m^{2}}{\beta^{2}} \int d x \cos \beta \hat{\phi} .
$$

The repulsive short-range interaction, characterized by the Luttinger parameter $K$, enters $\beta=\sqrt{2 \pi / K}$. For repulsive bosons $K \in[1, \infty]$. In the limit of small interactions [7] $(K \rightarrow \infty), K$ is connected to the LiebLiniger parameter $\gamma=m_{B} g / \hbar^{2} \rho_{0}$ via $K=\pi / \sqrt{\gamma}$ (where $g$ is the interaction strength, $m_{B}$ the mass of the bosons and $\rho_{0}$ the mean density). Furthermore, there are the sound velocity $v_{s}=v_{F} / K\left(\right.$ with $\left.v_{F}=\hbar \pi \rho_{0} / m_{B}\right)$, and the effective mass $m=\sqrt{2 t_{\perp} \rho_{0}} \beta$, where the tunnelamplitude $t_{\perp}$ between the condensates enters. The relevant length scale in the sine-Gordon model, determining the width of breathers and solitons, is set by $\sqrt{\hbar v_{s}} / m$. The sine-Gordon model should be a sound description of the system as long as $\sqrt{v_{s} \hbar} / m \gg \xi_{h} \sim 1 / \rho_{0} \sqrt{\gamma}$. Finally, the Josephson frequency is given by $\omega_{J}=m \sqrt{v_{s} / \hbar}$ [17], determining the period of the Josephson oscillations for $\beta \Phi_{0} \lesssim 1$. In the following, we set $v_{s}=\hbar=1$.

Experimental Realizability. - In the limit of weak interactions $(\beta \ll 1)$, the stiffness of the condensate becomes
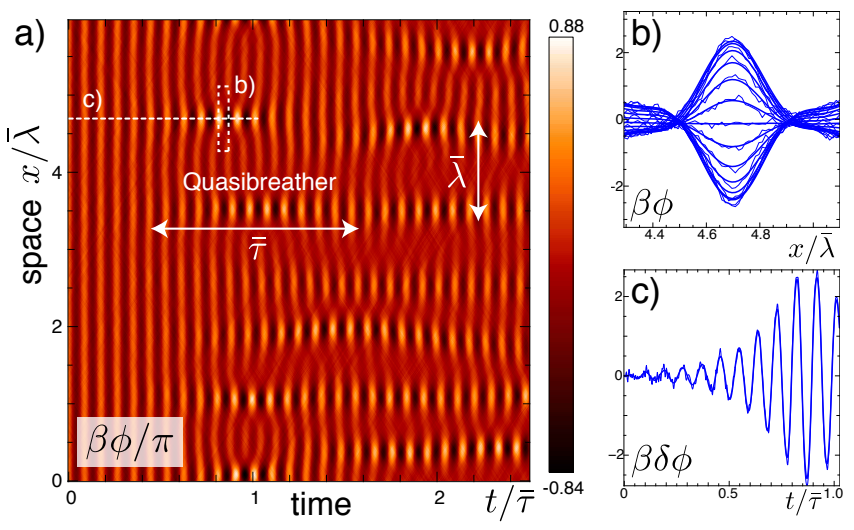

Figure 2. a) Space-time plot of the phase-field for a single simulation run. b) Spatial cut through the quasibreather highlighted in a) over one breather period (thin blue line; thick blue line: filtering out irrelevant short wavelength fluctuations). c) Temporal cut through the emerging QB taken at its center. $\delta \phi=\phi-\Phi$ is obtained by subtracting the spatially averaged field $\Phi=\frac{1}{L} \int_{0}^{L} d x \phi$. One observes the exponential amplification of initial quantum fluctuations. Here, $\beta \Phi_{0}=0.3 \pi$.

small. To avoid the breakdown of the hydrodynamical description, the mean condensate density $\rho_{0}$ must be large enough. In fact, the condition $\sqrt{v_{s} \hbar} / m \gg \xi_{h}$ translates into a lower bound for $\beta$ (and therefore into a lower bound for the interaction strength): $\beta^{2} \gg$ $4 \rho_{0}^{-1} \sqrt{t_{\perp} m_{\mathrm{B}}} / \hbar$. Within the present experimental setups the condensate density can be widely tuned. E.g., in [18] the $1 \mathrm{D}$ density of $\mathrm{Rb}^{87}$ atoms (with $m_{B}=1.44 \cdot 10^{-25} \mathrm{~kg}$ ) ranges from $\rho_{0}=3-100 \mu \mathrm{m}^{-1}$. For a transverse trap frequency $\omega_{\perp}=2 \pi \times 4 \mathrm{kHz}$, with $g=2 \hbar \omega_{\perp} a_{s}$ and $a_{s}=5.31 \cdot 10^{-9} \mathrm{~m}$, we have $K=9-52$ and therefore small values of the sine-Gordon parameter $\beta=\mathcal{O}\left(10^{-1}\right)$ are well achievable. Eventually, the tunnel-amplitude can be tuned between $t_{\perp} / \hbar=\left[\mathcal{O}\left(10^{2}\right)-\mathcal{O}\left(10^{4}\right)\right] / \mathrm{s}[5]$. Thus, for large densities $\rho_{0}=100 \mu \mathrm{m}^{-1}$ the lower bound on $\beta$ is $\mathcal{O}\left(10^{-1}\right)$ as well. In all numerical simulations, we use $\beta=0.1$. We conclude that after some fine-tuning of the experimental parameters, the proposed setup should be within reach.

Numerical results - Before the quench, the phase field $\phi$ is given by the offset $\Phi_{0}$ plus the small zero-point fluctuations of the free theory [Eq. (2) with $m=0$ ]. Within TWA, the fluctuations of the $\phi$ and $\Pi$ modes are initialized according to their Gaussian Wigner-distribution [16], omitting the $q=0$ mode.

This initial state should be experimentally achievable. One should start from the ground state at strong tunnel coupling $(m \gg 1)$, where the relative phase $\phi(x)=0$. During a slow linear rampdown of the tunnel-amplitude, all modes $\phi_{q \neq 0}$ will follow their time-dependent groundstate. It can be shown that the global phase $\Phi$ at the end of this process obeys $\beta^{2}\left\langle\Phi^{2}\right\rangle \sim 1 / \xi_{h} \rho_{0}$, which remains 
small as long as the number of bosons within the healing length is large. An additional potential tilt will produce a fixed phase offset $\Phi \simeq \Phi_{0}$. We will focus on rather small $\beta \Phi_{0} \lesssim 1$. For $\beta \Phi_{0} \rightarrow \pi$, in addition to breathers one observes the formation of solitons. Finally, we note that this 'phase-tuning' becomes impossible for infinite systems. This is due to the logarithmic divergence of phase fluctuations with system size $L$ in the massless groundstate, i.e., $\beta^{2}\left\langle\delta \hat{\phi}^{2}\right\rangle \sim \beta^{2} \ln L$. However, these are largely suppressed in the semiclassical limit $\beta \ll 1$. We always choose the system size such that the initial overall relative phase is well defined.

In Fig.2, the phase field for a single run of TWA is shown. At small times, the field is dominated by $\Phi(t)$ performing ordinary Josephson oscillations. At this point, the inhomogeneous part $\delta \phi(x, t)$ of $\phi=\Phi+\delta \phi$ can be treated as a small perturbation. The modes obey $\partial_{t}^{2} \phi_{k}+\left(k^{2}+m^{2} \cos [\beta \Phi(t)]\right) \phi_{k} \simeq 0$ for all $k \neq 0$, i.e., these are phonons with a periodically modulated mass. Modes with $|k| \in\left[0, m \sin \left|\beta \Phi_{0} / 2\right|\right]$ are parametrically amplified yielding $\phi_{k}(t) \sim e^{\Gamma_{k} t}$. The amplification rates $\Gamma_{k}$ for this linear regime (here displayed for $\beta \Phi_{0} \lesssim 1$ ) (see Fig. 3 a)

$$
2 \Gamma_{k} \simeq k \sqrt{\sin ^{2}\left(\beta \Phi_{0} / 2\right)-k^{2} / m^{2}},
$$

were found in [13, neglecting the damping of the driving $\Phi$-mode. After some time, nonlinear interactions between the amplified modes become important and lead to the formation of sharply localized oscillating structures (here denoted as 'quasibreathers'), which constitute the main phenomenon discussed in our paper. Once these localized oscillations get out of phase with respect to the background oscillations of $\Phi$, their energy is depleted again. One arrives at a steady-state, where QBs are randomly created and decay, with a typical lifetime $\bar{\tau}$ and a mean spatial distance $\bar{\lambda}$ (cf. Fig. 2). All these statistical quantities crucially depend on the initial value $\Phi_{0}$. It turns out that these localized modulations in the stochastic phase field can be connected to certain exact solutions of the SGM. These solutions, to be discussed in the following, are standing breathers riding on top of a homogeneous and oscillating background. We will demonstrate that this set of solutions is well suited to describe the numerical observations and provides analytical insight into the dependence of $\bar{\lambda}$ and $\bar{\tau}$ on $\Phi_{0}$.

Quasibreathers. - A Bäcklund transformation (see for instance [15]) allows to 'add' (anti)solitons to a given solution of the SGM, here taken to be the spatially homogeneous solution $\Phi(t)$ with $\Phi(0)=\Phi_{0}$ and $\dot{\Phi}(0)=0$. Adapting the approach of [15], we obtain quasibreathersolutions by adding a (standing) soliton and the corresponding antisoliton to $\Phi$. A standing SGM-breather is characterized by its amplitude (e.g., [19]). The solutions here depend in addition on the amplitude of the underlying background oscillations $\Phi_{0}$ (furthermore, there is a minor dependence on the precise value of the relative a)
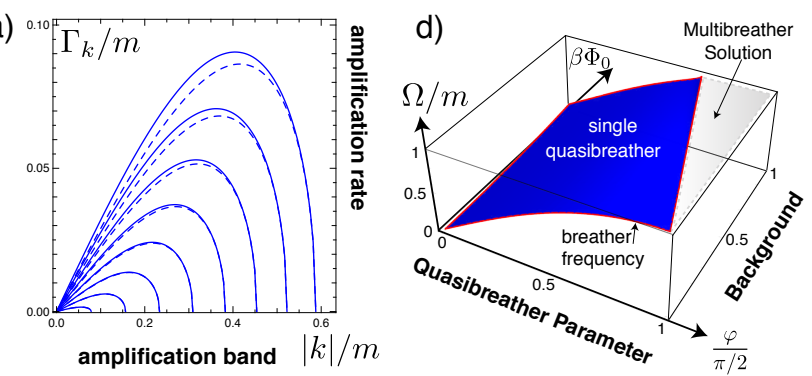

b)
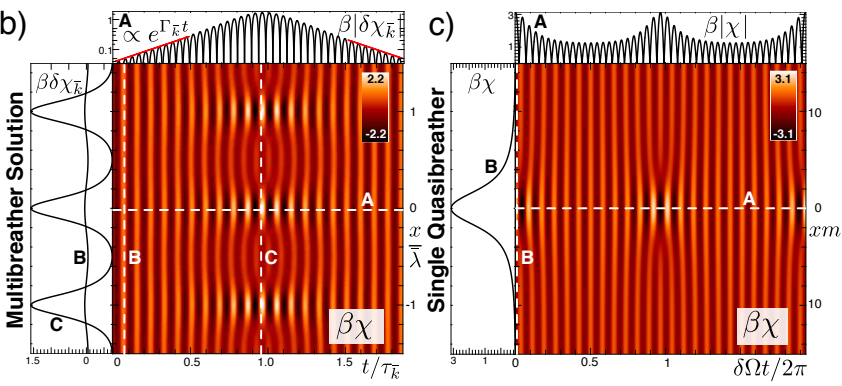

Figure 3. Phononic zero-point fluctuations are amplified parametrically driven by the overall phase $\Phi$ performing Josephson oscillations. a) Corresponding amplification rate $\Gamma_{k}$ of phonons with wavenumber $k$ for $\beta \Phi_{0} / \pi \in 0.05-0.4$ according to Eq. 3) (dashed line). Solid lines show the rate obtained from the 'multibreather' solution Eq. (5). b) Plot of a (spatially periodic) 'multibreather' solution reducing to a driven phonon with wavenumber $k=m \beta \Phi_{0} / \sqrt{8}$ for $t / \tau_{\bar{k}} \rightarrow-\infty$. Cut A shows the parametric amplification of this phonon (B) driven by the oscillating background (we plot $\delta \chi_{\bar{k}}$, substracting this background). They provide 'seeds' for the formation of Quasibreathers (C) with a lifetime $\tau_{\bar{k}}$. Here, $\beta \Phi_{0}=0.3 \pi$. c) Plot of a 'single quasibreather' (periodic in time) with $\beta \Phi_{0}=0.3 \pi$ and $\varphi=1.02$. d) Mean frequency $\Omega \equiv m-\delta \Omega$ of a 'single quasibreather' $\left(|\sin \varphi|<\left|\cos \frac{\beta \Phi_{0}}{2}\right|\right)$ depending on the amplitude of the background oscillations and the parameter $\varphi$. For $\Phi_{0}=0$, one restores the unperturbed breather frequency $\Omega=m \sin \varphi$.

phase $\Delta_{0}$ between breather and background at $t=0$ ). They have the form

$$
\chi(x, t)=\frac{4}{\beta} \arctan \left[\mathcal{G}\left(x, t ; \Phi_{0}, \Delta_{0}, \varphi\right)\right]+\Phi(t) .
$$

For $\Phi_{0}=0$, the parameter $\varphi \in[0, \pi / 2]$ determines the unperturbed breather frequency $m \sin \varphi$ and $\max \chi=$ $\beta^{-1}(2 \pi-4 \varphi)$. An explicit expression for $\mathcal{G}$ and a detailed discussion of Eq. (4) are given in the supplement. Here, we focus on the most relevant features in the limit $\beta \Phi_{0} \lesssim 1$

The central observation is that by placing a breather on top of the oscillating background, its amplitude becomes time-dependent (see Figs.3b,c). The background $\Phi(t)$ amplifies the breather while the frequency of the latter decreases. This is due to the fact that the effective curvature of the cosine-potential decreases for larger field amplitudes. Eventually, both run out of phase and, 
subsequently, the breather gets damped. The relative phase-drift occurs at a frequency

$$
\delta \Omega\left(\varphi, \Phi_{0}\right)=\frac{m \sqrt{\left|\cos ^{2}\left(\beta \Phi_{0} / 2\right)-\sin ^{2} \varphi\right|} \cos \varphi}{1+\sin \varphi} .
$$

As long as $\sin \varphi<\cos \left(\beta \Phi_{0} / 2\right), \chi$ describes a single breather whose amplitude is modulated with a period $2 \pi / \delta \Omega$ (Fig. 3c). This constitutes a stable QB solution of the SGM. As the background oscillates at frequency $m$, one can understand $\Omega \equiv m-\delta \Omega$ as the mean frequency of the quasibreather (Fig. $3 \mathrm{~d}$ ).

For $\sin \varphi \rightarrow \cos \left(\beta \Phi_{0} / 2\right)$, however, the period diverges. In fact, it turns out that for $\sin \varphi>\cos \left(\beta \Phi_{0} / 2\right), \chi$ is periodic in space rather than in time (Fig. $3 \mathrm{p}$ ). It describes a set of quasibreathers at distance $2 \pi / k=$ $2 \pi m^{-1}\left|\cos ^{2}\left(\beta \Phi_{0} / 2\right)-\sin ^{2} \varphi\right|^{-1 / 2}$.

These 'multibreather' solutions have remarkable properties. For $t \rightarrow-\infty$, they reduce to a phonon $\delta \chi_{k}$ with wavenumber $k$, superimposed on the oscillating background. It is amplified parametrically, yielding

$$
\delta \chi_{k} \propto e^{\Gamma_{k} t} \zeta(t) \cos k x
$$

where $\zeta(t)$ is a periodic function and the amplification rate $\Gamma_{k} \equiv \delta \Omega\left(\varphi(k), \Phi_{0}\right)$. This rate is in agreement with the results in Eq. (3) for small $\beta \Phi_{0}$. At later times, the characteristic breather peaks form (Fig. 3r), exist during a time set by $\tau_{k}=4 / \Gamma_{k}$ and decay again for $t \rightarrow \infty$. Note that $\chi$ therefore describes the prototypical formation of QBs out of fluctuations in a single mode $\phi_{k}$.

Our numerical analysis shows (see below) that one can infer the properties of typical QBs observed within the stochastic TWA from these 'ideal' solutions $\chi$. In a given run, zero-point fluctuations in all modes are present. However, in the weakly interacting limit $(\beta \ll 1)$, the parametric instability automatically filters out modes with $k \approx \bar{k}$, where $\bar{k} \simeq m \beta \Phi_{0} / \sqrt{8}$ denotes the maximally amplified mode. Thus, the typical distance between QBs is roughly given by $\bar{\lambda}=2 \pi / \bar{k}$. Although the strictly periodic 'multibreather' solution $\chi$ is not directly observed, individual QBs are well described by $\chi$ as long as their amplitude is large enough compared to the noisy background. Typical QBs decay after a lifetime $\bar{\tau} \equiv \tau_{\bar{k}}$ (cf. Fig. 3k). When their amplitude is depleted down to the noise level, the exact solution ceases to be relevant. Then the background oscillations initiate the amplification process again, leading to the formation of new QBs. This explains the observed stochastic creation and annihilation of QBs at large times $\Gamma_{\bar{k}} t \gg 1$.

Statistical Analysis. - These predictions agree well with the statistical analysis of the quench within TWA. For every run, we numerically track all QBs, showing their shape $\phi_{\mathrm{QB}}(x)$ at the time of maximum amplitude (Fig.44). A comparison of the mean QB-shape $\left\langle\phi_{\mathrm{QB}}\right\rangle$ and $\chi$ (with $k=\bar{k}$ ) shows excellent agreement. Note that no fit parameter enters here.

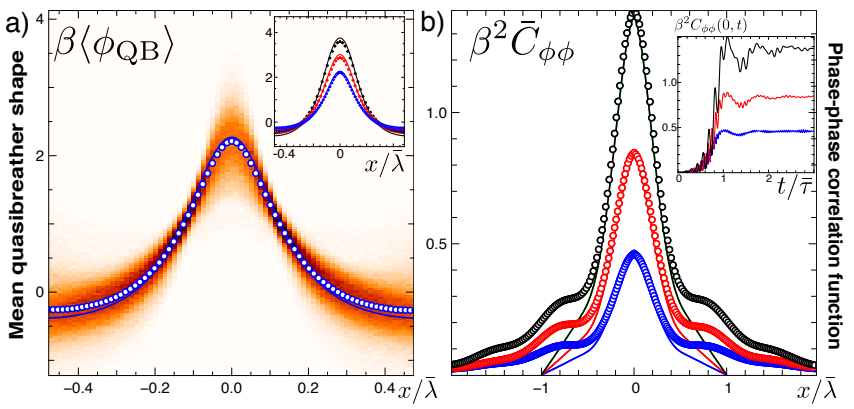

Figure 4. a) Mean shape of QBs taken at their maximal value, obtained from TWA (blue dots) and full distribution of shapes (color plot). We track QBs numerically run-byrun in the interval $t / \bar{\tau} \in[0.5,4]$ for $\beta \Phi_{0} / \pi=0.3$. Comparison to the analytical solution $\chi(x, 0)$ with $\bar{k}$ (blue, solid line; $\Delta_{0}=0$ ) shows almost perfect agreement. Inset) Various $\beta \Phi_{0} / \pi=0.3,0.4,0.5$ (from bottom to top). No fitparameter enters. b) Correlation function $C_{\phi \phi}$, averaged over time $t / \bar{\tau} \in[2.25,3]$ (dots) with $\beta \Phi_{0} / \pi=0.3,0.4,0.5$ (from bottom to top). Locally, it agrees well with Eq. (7). Inset shows $C_{\phi \phi}(0, t)$, demonstrating that the field enters a steady state for $t / \bar{\tau} \gtrsim 1$.

Finally, Fig. $4 \mathrm{~b}$ shows the equal-time correlation function $C_{\phi \phi}(x, t)=\langle\hat{\phi}(x, t) \hat{\phi}(0, t)\rangle-\langle\hat{\phi}(0, t)\rangle^{2}$ evaluated with TWA. For $t / \bar{\tau}>1$, it saturates, indicating that the field enters a statistical steady state. One can find a decent approximation for $C_{\phi \phi}$ at large times $t \gg \bar{\tau}$, assuming that $\phi(x, t)$ can be represented as a sum of independent QBs, at an average density $1 / \bar{\lambda} \bar{\tau}$ in the $(x, t)$-plane:

$$
C_{\phi \phi} \approx \int_{-\bar{\lambda} / 2}^{\bar{\lambda} / 2} \frac{d x_{0}}{\bar{\lambda}} \int_{-\bar{\tau} / 2}^{\bar{\tau} / 2} \frac{d t_{0}}{\bar{\tau}} \tilde{\chi}\left(x ; x_{0}, t_{0}\right) \tilde{\chi}\left(0 ; x_{0}, t_{0}\right),(7)
$$

which describes the core part of $C_{\phi \phi}$ fairly well (cf. Fig. 4). Here, a single QB from the 'multibreather' solution centered at $x_{0}$ enters: $\tilde{\chi}\left(x ; x_{0}, t_{0}\right)=\Theta\left(\frac{\bar{\lambda}}{2}-\mid x-\right.$ $\left.x_{0} \mid\right) \chi\left(x-x_{0}, t_{0} ; \bar{k}\right)(\Theta$ denotes the heavy-side step function).

This correlation function is directly accessible in experiments and should distinctively reveal the presence of QBs. Moreover, one could simply perform a direct statistical analysis of $\phi$ (cf. Fig.44).

Summary. - We predict the formation of localized modulations in the relative phase field, after suddenly switching on the tunnel-coupling between a pair of quasi1D condensates. These 'Quasibreathers' grow out of initial quantum fluctuations, mimicking processes that are crucially important in other areas like cosmology. They can be well described by exact analytical solutions of the sine-Gordon model. We derived their mean lifetime and density after the system reaches a statistical steady state. These predicitions are consistent with our numerical simulations. An experimental realization, even with present setups seems to be within reach.

Acknowledgements. - We thank J. Schmiedmayer for 
fruitful discussions. Financial support by the EmmyNoether program and the SFB/TR 12 is gratefully acknowledged. CN gratefully acknowledges the hospitality of Boston University, where parts of this work were performed.

* clemens.neuenhahn@physik.uni-erlangen.de

[1] M. Gleiser and R. C. Howell, Phys. Rev. E 68, 065203 (2003).

[2] M. A. Amin, R. Easther, H. Finkel, R. Flauger, and M. P. Hertzberg, arXiv:1106.3335v1 (2011).

[3] M. A. Amin, R. Easther, and H. Finkel, Journal of Cosmology and Astroparticle Physics 12 (2010).

[4] E. Farhi, N. Graham, A. H. Guth, N. Iqbal, R. R. Rosales, and N. Stamatopoulos, Phys. Rev. D 77, 085019 (2008).

[5] S. Hofferberth, I. Lesanovsky, B. Fischer, T. Schumm, and J. Schmiedmayer, Nature 449, 324 (2007).

[6] T. Betz, S. Manz, R. Bücker, T. Berrada, C. Koller, G. Kazakov, I. E. Mazets, H.-P. Stimming, A. Perrin, T. Schumm, and J. Schmiedmayer, Phys. Rev. Lett. 106, 020407 (2011).

[7] V. Gritsev, A. Polkovnikov, and E. Demler, Phys. Rev. B 75, 174511 (2007).

[8] V. Gritsev, E. Demler, M. Lukin, and A. Polkovnikov, Phys. Rev. Lett. 99, 200404 (2007).

[9] C. De Grandi, R. A. Barankov, and A. Polkovnikov, Phys. Rev. Lett. 101, 230402 (2008).

[10] J. Lancaster, E. Gull, and A. Mitra, Phys. Rev. B 82, 235124 (2010).

[11] A. Iucci and M. A. Cazalilla, New Journal of Physics 12, 055019 (2010).

[12] M. S. Foster, E. A. Yuzbashyan, and B. L. Altshuler, Phys. Rev. Lett. 105, 135701 (2010).

[13] P. B. Greene, L. Kofman, and A. A. Starobinsky, Nuclear Physics B , 423 (1999).

[14] I. Bouchoule, Eur. Phys. J. D , 147 (2005).

[15] D. W. McLaughlin and A. C. Scott, Phys. Rev. A 18, 1652 (1978).

[16] A. Polkovnikov, Annals of Phys. 325 (2010).

[17] N. K. Whitlock and I. Bouchoule, Phys. Rev. A 68, 053609 (2003)

[18] P. Krüger, S. Hofferberth, I. E. Mazets, I. Lesanovsky, and J. Schmiedmayer, Phys. Rev. Lett. 105, 265302 (2010).

[19] K. Maki and H. Takayama, Phys. Rev. B 20, 5002 (1979). 


\section{Supplementary Material}

Derivation of Quasibreather Solution - Here, we sketch the crucial steps involved in the derivation of the quasibreather solutions discussed in the main text. The details of the rather lengthy calculation will be shown elsewhere.

In general, the Bäcklund transform allows for the construction of arbitrary multisoliton waves for the classical sine-Gordon equation (SGE) $\phi_{t t}-\phi_{x x}+\sin \phi=0$ (setting for the moment $\beta=m=1$ ). The idea is that starting from an arbitrary solution $\psi$ of the SGE, one can easily show that the field $\phi$ fulfilling the Bäcklund equations (BE's)

$$
\begin{aligned}
& \frac{1}{2} \frac{\partial}{\partial z}(\phi-\psi)=\alpha \sin \left[\frac{\phi+\psi}{2}\right], \\
& \frac{1}{2} \frac{\partial}{\partial \tau}(\phi+\psi)=\frac{1}{\alpha} \sin \left[\frac{\phi-\psi}{2}\right],
\end{aligned}
$$

where $\alpha \in \mathbb{C}$ is an arbitary constant, $z \equiv \frac{x-t}{2}$ and $\tau \equiv \frac{x+t}{2}$, is a solution as well. Furthermore, it turns out that $\phi$ obtained in this manner is just the original solution plus an additional soliton (see for instance [15]). For real values of $\alpha$, the velocity of the added soliton is given by $v=\frac{\alpha^{2}-1}{\alpha^{2}+1}$. In the considered case, the known solution $\psi$ is the homogeneous part of the phase-field, $\Phi(t)$, performing Josephson oscillations according to $\ddot{\Phi}=-\sin \Phi$. As the numerical analysis with the TWA shows, slight inhomogeneties in the field are amplified parametrically, eventually leading to the formation of (standing) breather-like excitations, which have a finite lifetime. We construct exact solutions of the SGE describing standing breathers on top of this oscillating 'background'. As it was shown in the main publication, it turns out that the solutions constructed here are well suited to describe the observed localized patterns in the field $\phi$.

In the absence of the background, ordinary breathers of the SGE can be obtained by adding a soliton and an antisoliton to the 'vaccum'. Here, we follow closely this strategy. For the purpose of creating a single soliton, the parameter $\alpha$ can be choosen to be real. However, in order to create finally a breather out of a soliton-antisoliton pair, we must allow for $\alpha \in \mathbb{C}$. Being interested in standing quasibreathers (breather + background), it turns out that in a first step, formally, one has to add a single 'soliton' with $|\alpha|=1$ on top of the background. Solving Eqs. 89) with $\psi \equiv \Phi$, one obtains a set of solutions $\phi_{\alpha}(x, t)$. Fortunately, for the second step (adding the corresponding anti-soliton $\phi_{\beta}$ ), one can use the fact [15] that

$$
\chi(x, t)=4 \arctan \left[\frac{\alpha+\beta}{\alpha-\beta} \tan \left(\frac{\phi_{\alpha}-\phi_{\beta}}{4}\right)\right]+\Phi(t)(10)
$$

is a solution of the SGE as well, as long as both $\phi_{\alpha}$ and $\phi_{\beta}$ were obtained from Eqs. 89 with $\psi \equiv \Phi$. The first step (finding the solution for a 'soliton' on top of an oscillating background) involves solving the set of coupled differential equations Eqs. (89). The second step mainly consists in matching $\alpha$ and $\beta$ properly (it turns out that $\alpha=\beta^{*}$.

Finally, one finds that the quasibreather solutions $\chi$ have the form

$$
\chi=\frac{4}{\beta} \arctan \left(\frac{2}{\tan \varphi} \frac{\operatorname{Im} \mathcal{F}\left[x, t ; \Phi_{0}, \Delta_{0}, \varphi\right]}{1+|\mathcal{F}|^{2}}\right)+\Phi .
$$

The argument of the arctan-function corresponds to the function $\mathcal{G}$ introduced in the main text. In fact, there are two sets of solutions:

$$
\frac{\mathcal{F}}{f}=\left\{\begin{array}{cl}
\frac{\tanh \left[\frac{m f x}{4}+\mathcal{C}+i \Delta\right]+\mathcal{A}}{\mathcal{B}}, & \cos \varphi>\sin \left(\frac{\beta \Phi_{0}}{2}\right) \\
\frac{-\tan \left[\frac{m f x}{4}+\mathcal{C}+i \Delta\right]+\mathcal{A}}{\mathcal{B}}, & \cos \varphi \leq \sin \left(\frac{\beta \Phi_{0}}{2}\right)
\end{array}\right.
$$

Whereas the well known, standing SGM-breather is characterized by a single parameter (its amplitude), the solution here depends on three parameters. The amplitude of the underlying background oscillations $\Phi_{0}$, the maximal amplitude of the breather (determined by a parameter $\varphi \in[0, \pi / 2])$, and the relative phase between breather and background oscillations, set by $\Delta_{0}$.

The function $\Delta(t)$ in Eq. 12 grows monotonically during the temporal evolution of $\chi$. It is given by the integral equation

$$
\Delta(t)=\frac{m f \sin (2 \varphi)}{2} \int_{0}^{t} d t^{\prime} \frac{\sin ^{2}\left[\beta \Phi\left(t^{\prime}\right) / 2\right]}{|\mathcal{B}|^{2}\left(t^{\prime}\right)}+\Delta_{0} .
$$

Furthermore, $\mathcal{B}(t) \equiv \beta m^{-1} \dot{\Phi}+2 i \sin \varphi \sin (\beta \Phi / 2)$ and $\mathcal{A}(t) \equiv 2 f^{-1} \cos \varphi \cos (\beta \Phi(t) / 2)$, where $f \equiv 2 \mid \cos ^{2} \varphi-$ $\left.\sin ^{2}\left(\beta \Phi_{0} / 2\right)\right|^{1 / 2}$. The solutions for $\cos \varphi>\sin \left(\beta \Phi_{0} / 2\right)$ are periodic in time [with $\left.\mathcal{C}(t)=\tanh ^{-1}\left(\sqrt{\mathcal{A}^{2}-1}-\mathcal{A}\right)\right]$. These are termed 'single quasibreathers' and denoted by $\chi_{\varphi}$. For $\cos \varphi \leq \sin \left(\beta \Phi_{0} / 2\right), \chi$ is periodic in space. These 'multibreather' solutions $\chi_{k}$ are labeled by the wavenumber $k=m f / 2$ [in this case $\mathcal{C}(t)=\tan ^{-1}\left(\sqrt{\mathcal{A}^{2}+1}+\mathcal{A}\right)$ ].

From now on, we consider $\beta \Phi_{0} \lesssim 1$. Averaging $d \Delta / d t$ over one Josephson period, one obtains $2 \Delta \simeq \delta \Omega t+2 \Delta_{0}$, with a frequency difference (see main text)

$$
\delta \Omega\left(\varphi, \Phi_{0}\right)=\frac{m f \cos \varphi}{2(1+\sin \varphi)} .
$$

The 'multibreather' solution $\chi_{k}$ has remarkable properties (see Fig. 5). For $t \rightarrow-\infty[\Delta \rightarrow-\infty]$ it reduces to a phonon $\delta \chi_{k}$ with wavenumber $k$, superimposed on the oscillating background. It is amplified parametrically, yielding

$$
\delta \chi_{k} \propto e^{\Gamma_{k} t} \zeta(t) \cos k x,
$$

where $\zeta(t)$ is a periodic function. The amplification rate $\Gamma_{k}=\delta \Omega\left(\varphi(k), \Phi_{0}\right)$. As soon as $\Delta \simeq-1$, the characteristic breather peaks form and exist during a time set by $\tau_{k}=4 / \Gamma_{k}$. The maximum is reached for $\Delta \simeq 0$, 

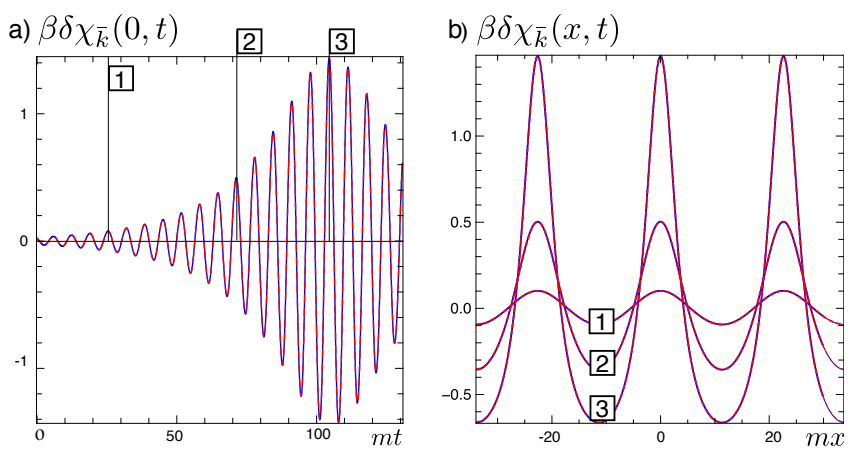

Figure 5. a) 'Multibreather' solution $\chi_{k}$ with $k=m \beta \Phi_{0} / \sqrt{8}$ and $\beta \Phi_{0}=0.25 \pi$, evaluated at the center of the excitation at $x=0$. We plot $\delta \chi_{k}=\chi_{k}-L^{-1} \int_{0}^{L} d x \chi_{k}$ ( $L$ denotes the system size). The inhomogenity $\delta \phi$ is amplified parametrically. The blue, solid lines show the analytical result. b) Cut in $x$-direction for the times indicated in a). One observes the transition from initial, sinusoidal inhomogenities into the characteristic breather-peaks at large times. The analytical solution $\chi_{k}$ is checked against a direct simulation of the sine-Gordon equation with a 4th-order Runge-Kutta algorithm (with appropriate initial conditions; red, dashed line)

where one observes a set of QBs with distance $2 \pi / k$. For $\Delta \rightarrow \infty$, these decay again. In constrast, $\chi_{\varphi}$ describes a single QB whose amplitude is modulated quasiperiodically with period $2 \pi / \delta \Omega\left(\varphi, \Phi_{0}\right)$.

Correlation Function. -At long times $t / \bar{\tau} \gg 1$, as reported in the main text, one observes a steady state characterized by the perpetual creation and annihilation of breathers. In order to confirm the presence of quasibreathers in the experiment, we propose to evaluate the phase-phase correlation function $C_{\phi \phi}(x) \equiv$ $\langle\hat{\phi}(x, t) \hat{\phi}(0, t)\rangle-\langle\hat{\phi}(x, t)\rangle\langle\hat{\phi}(0, t)\rangle$. Note that as we restrict to initial detunings $\beta \Phi_{0} \lesssim 1$, we do not observe the creation of solitons after the quench and the corresponding phase slips in $\phi$. Therefore, we do not have to switch to correlation functions like $\langle\cos \beta \hat{\phi}(x, t) \cos \beta \hat{\phi}(x, 0)\rangle$.

One can find a decent analytical approximation for $C_{\phi \phi}$ based on the 'multibreather' solutions discussed above. The 'multibreather' solution is strictly periodic in space. However, note that this exact solution of the classical sine-Gordon is only valid when the breather amplitude is large enough compared to the noisy background. We assume that each of these quasibreathers centered at $x_{i}$ (their phases are encoded in the time $t_{i}$ ) is described by

$$
\tilde{\chi}\left(x ; x_{i}, t_{i}\right) \equiv \Theta\left(\frac{\bar{\lambda}}{2}-\left|x-x_{i}\right|\right) \Theta\left(\frac{\bar{\tau}}{2}-\left|t_{i}\right|\right) \chi_{\bar{k}}\left(x-x_{i}, t_{i}(i) 6\right)
$$

Here, $\chi_{\bar{k}}$ denotes the 'multibreather' solution with $k=\bar{k}$ and $\Theta$ is the heavy-side step function. For simplicity, we pick the special set of solutions with $\Delta_{0}=0$ (at small values of $\beta \Phi_{0}, \chi_{\bar{k}}$ depends only slightly on the precise relative phase between breather and background, $\Delta_{0}$, anyway). With this choice of $\Delta_{0}$, the 'multibreather' solution $\chi_{\bar{k}}(x, t)$ reaches its maximal value at $t=0$ and $x=n \bar{\lambda}$ with $n \in \mathbb{Z}$. After some time, a 'real' quasibreather dives into the noisy background and the 'multibreather' solution ceases to be valid. While it is hard to determine the precise time when this happens, in a reasonable approximation, we cut-off $\tilde{\chi}\left(x ; x_{i}, t_{i}\right)$ for $\left|t_{i}\right|>\bar{\tau} / 2$. Here, $\bar{\tau}$ is the 'lifetime' of the 'multibreather' solution (see main text).

In a given experimental run $\alpha, \phi_{\alpha}(x, t) \simeq$ $\sum_{j=1}^{n_{\alpha}} \tilde{\chi}\left(x ; x_{i}^{\alpha}, t_{i}^{\alpha}\right)$ with $n_{\alpha}$ denoting the number of breathers at time $t$ (neglecting short-wavelength radiation). It is understood that the distance between neighboring breathers $\left|x_{i}-x_{i+1}\right| \simeq \bar{\lambda}$. Under the assumption of independent quasibreathers and with $\langle\hat{\phi}\rangle \simeq 0$ as well as $C_{\phi \phi}(x, t) \simeq C_{\phi \phi}(x)$ for $t / \bar{\tau} \gg 1$, one obtains

$$
C_{\phi \phi}(x) \simeq \overline{\sum_{i=1}^{n_{\alpha}} \tilde{\chi}\left(x ; x_{i}^{\alpha}, t_{i}^{\alpha}\right) \tilde{\chi}\left(0 ; x_{i}^{\alpha}, t_{i}^{\alpha}\right) .}
$$

The bar denotes the average over experimental runs. Note that only a single quasibreather with $\left|x_{i_{0}}^{\alpha}\right| \leq \bar{\lambda} / 2$ contributes to the average and that the correlation function vanishes in this approximation of independent breathers for $|x|>\bar{\lambda}$. Therefore, we can replace the average over runs by an average over $x_{0} \equiv x_{i_{0}}^{\alpha}$ and $t_{0} \equiv t_{i_{0}}^{\alpha}$ yielding

$$
\begin{aligned}
& C_{\phi \phi}(x) \\
\simeq & \frac{1}{\bar{\tau} \bar{\lambda}} \int_{-\bar{\lambda} / 2}^{\bar{\lambda} / 2} d x_{0} \int_{-\bar{\tau} / 2}^{\bar{\tau} / 2} d t \tilde{\chi}\left(x ; x_{0}, t_{0}\right) \tilde{\chi}\left(0 ; x_{0}, t_{0}\right),
\end{aligned}
$$

which can be evaluated numerically. 